Table 2 shows that pure aragonite was the only type of calcium carbonate to precipitate from sea-water by $\mathrm{CO}_{2}$ extraction. This finding has also been made by other workers ${ }^{2}$. Furthermore, the rate of crystallization of aragonite in these experiments was so slow (even at the unnaturally high supersaturation value achieved by adding sodium carbonate to make the solution $p \mathrm{H} \mathrm{9)}$ that such crystallization can scarcely be used to explain the rapid formation of whitings seen in the sea off Doha.

Koninklijke/Shell Exploratie

en Produktie Laboratorium,

Rijswijk, The Netherlands.

1 Wells, A. J., and Illing, L. V., in Developments in Sedimentology, 1, 429, 2 Simkiss, K., Nature, 201, 492 (1964).

\section{An Experimental Determination of the Photo-ionization Cross-section of Atomic Hydrogen}

Arthough many theoretical calculations of the photoionization cross-section of atomic hydrogen have been performed (see review by Bates ${ }^{1}$ ), no experimental determination of this quantity has previously been made. An experiment is being carried out here which has yielded a value for the photo-ionization cross-section of atomic hydrogen at $850 \cdot 6 \AA$.

Atomic hydrogen is produced by flowing molecular hydrogen through a discharge tube in which an intense radio frequency discharge is run. This dissociates a large fraction of the hydrogen molecules. The mixture of atoms and molecules is then flowed quickly through an all-glass absorption cell which is attached to the exit slit of a onemetre normal-incidence vacuum monochromator. Radiation emerging from the exit slit passes down the absorption cell and is detected by a sensitized photomultiplier placed at the far end of the cell. The light source used to illuminate the entrance slit of the monochromator is similar to that described by Garton ${ }^{2}$ and consists essentially of a short-duration high-current spark prcduced by discharging a capacitor bank. The light source is filled with low-pressure argon gas and the radiation produced by it consists mainly of emission lines of neutral and multiply ionized argon.

The intensity of the radiation emerging from the absorption cell is measured under three conditions: (i) intensity $I_{0}$, with the absorption cell evacuated; (ii) $I_{1}$, with the absorption cell filled with molecular hydrogen at pressure $p_{1}$; (iii) $I_{2}$, with partially dissociated hydrogen in the absorption cell at total pressure $p_{2}$. The degree of dissociation $\alpha$ is determined by the Wrede Harteck method ${ }^{3}$.

From (i) and (ii) the photo-absorption cross-section of molecular hydrogen, $\sigma_{M}$, can be determined from the equation:

$$
I_{1}=I_{0} \exp \left(-N \sigma_{M} \frac{p_{1}}{760} \frac{273}{T} l\right)
$$

where $N$ is Loschmidt's number, $2.69 \times 10^{19}$ particles/ c.c., $T$ is the absolute temperature of the gas $\left({ }^{\circ} \mathrm{K}\right)$, and $l$ is the length of the absorption cell (cm).

From a knowledge of $\alpha$ and $p_{2}$, the partial pressures $p_{A}$, $p_{M}$ of atomic and molecular hydrogen can be calculated. Thus, using (i) and (iii) and the result for $\sigma_{M}$ derived from (1), the photo-ionization cross-section of atomic hydrogen, $\sigma_{A}$, can be found from the equation:

$$
I_{2}=I_{0} \exp \left(-\left\{N \sigma_{A} \frac{P_{A}}{760} \frac{273}{T}+N \sigma_{M} \frac{P_{M}}{760} \frac{273}{T}\right\} l\right)
$$

$p_{1}, p_{2}$ and $\alpha$ are measured at each end of the absorption cell and a correction for the concentration gradient along the length of the cell is applied.
Due to the difficulty in obtaining sufficient intensity at the photomultiplier to make reliable measurements, initial results have been confined to a wave-length, $850.6 \AA$, which corresponds to a strong emission line of the light source and a very small absorption cross-section of molecular hydrogen.

The mean value of the molecular cross-section (together with the probable error) at $850.6 \AA$ is $(0.213 \pm 0.010) \times$ $10^{-18} \mathrm{~cm}^{2}$, which is not in agreement with Cook and Metzger's ${ }^{4}$ result of $\sim 3.2 \times 10^{-18} \mathrm{~cm}^{2}$ at this wave-length. This may be due to a difference in the effective resolution of the two systems; as there is no continuous absorption in this region the measured value of $\sigma_{M_{2}}$ will depend critically on the resolution. Cook and Metzger used a continuum light source in conjunction with a monochromator with a pass band of $0.5 \AA$. Although our instrument has a similar bandpass, we used a line source and, as the width of the $850.6 \AA A$ IV line is probably of the order of or less than $0.1 \AA$, our resolution is much better than the bandpass of the instrument.

The mean value of the photo-ionization cross-section of atomic hydrogen at $850 \cdot 6 \AA$ is:

$$
(5 \cdot 15 \pm 0 \cdot 18) \times 10^{-18} \mathrm{~cm}^{2}
$$

This is in good agreement with the most recently available theoretical value which is given in graphical form by Bates'. At $850 \cdot 6 \AA$ the theoretical value lies between $5 \cdot 1$ and $5 \cdot 2 \times 10^{-18} \mathrm{~cm}^{2}$.

This work was initiated by Dr. R. B. Cairns and I thank him for his help during the early stages.

$$
\text { J. D. E. BEYNON }
$$

Radio and Space Research Station,

Ditton Park, Slough, Bucks.

'Bates, D. R., Atomic and Molecular Processes (Academic Press, New York, 1962).

${ }^{2}$ Garton, W. R. S., Jn. Sei. Instr. 36, 11 (1959).

${ }^{3}$ Greaves, J. C., and Linnett, J. W., Trans. Farad. Soc., 55, Pt. 8 (August 1959).

4 Cook, G. R., and Metzger, P. H., J. Opt. Soc. Amer., 54, 968 (1964).

\section{Structure of Catena-di $\mu$-Fluorodifluoro-} diaquohafnium(IV) monohydrate $\left(\mathrm{HfF}_{4} \cdot 3 \mathrm{H}_{2} \mathrm{O}\right)$

Altнough the trihydrate of hafnium tetrafluoride and its dehydration products are stoichiometrically similar to the corresponding compounds of zirconium, it has been shown ${ }^{1}$ that they are not isostructural. In general, compounds of the two elements do show identical structure (for example, the anhydrous fluorides ${ }^{2}$ ), and the fluoride hydrates must be particularly susceptible to differences in bond energy. The structure of $\mathrm{ZrF}_{4} \cdot 3 \mathrm{H}_{2} \mathrm{O}$ has been reported previously ${ }^{3}$, and the hafnium analogue has now been investigated.

Crystals were prepared, as previously described ${ }^{1}$, as an apparently homogeneous sample of colourless needles. Single crystal studies have, however, demonstrated the existence of two distinct species. One of these is monoclinic, $a=25 \cdot 48, b=7 \cdot 5 \mathrm{l}, c=23.02 \AA, \beta=150^{\circ}, 16$ molecules per unit cell. Reflexions are systematically absent for all $h k l$ when $h+k$ is odd, and for $h k l$ with $h$ even, when $k / 2+1$ is odd. Reflexions with $k$ odd are weak and are markedly diffused along $c^{*}$, thus suggesting that the structure is at least partially disordered. Our investigation has not proceeded further than the location of the hafnium atom positions.

The other modification is also monocinic, with $a=6 \cdot 70$, $b=10.55, c=7.71 \AA, \beta=94^{\circ}, 4$ molecules per unit cell, space group $P 2_{i, c}$. The structure of these crystals had no such eomplications and has been solved by conventional methods. The current reliability factor, assuming isotropic thermal motion, is $0 \cdot 14$.

All light atoms were assumed to be fluorine for the purpose of structure factor calculation. The peak electron densities on a subsequent Fourier synthesis were 11.6, $10 \cdot 7,9 \cdot 9,9 \cdot 4,8 \cdot 6,6 \cdot 7$ and $6 \cdot 5$ electrons $\AA^{-3}$. The smallest 